\title{
Workforce Development for Ultra-High Performance Concrete
}

\author{
Grace McMurry, Brad Weldon*, and Craig Newtson \\ Department of Civil Engineering, New Mexico State University, Las Cruces, NM 88003-8001
}

\begin{abstract}
Ultra-high performance concrete (UHPC) is a cementitious material with a dense microstructure that contributes to high compressive strengths as well as enhanced durability properties. UHPC also possesses significant post-cracking strength and ductility due to the addition of fibers. These characteristics produce a material that provides advantages over conventional concrete; however, high costs attributed to materials and production, lack of industry familiarity and knowledge, and the absence of standardized design procedures have impeded its wide-spread use. To help disseminate knowledge on UHPC, the first of two workforce development symposiums on UHPC was held on April 17 - 18, 2018 in Las Cruces, New Mexico. The symposium consisted of presentations and hands-on demonstrations to introduce UHPC and distribute the findings of almost a decade of research conducted in New Mexico to a diverse audience including members of the New Mexico Department of Transportation, contractors, designers, researchers, and concrete suppliers.
\end{abstract}

\section{Introduction}

\subsection{UHPC background}

Ultra-high performance concrete (UHPC) is a fiber reinforced composite concrete material that is known for having greater compressive strengths, tensile strengths, durability, corrosion resistance, post-cracking capacity than conventional concrete. UHPC is made using a high content of cementitious materials, a low water-to-cement ratio, silica fume, high-range water reducing admixtures (HRWRA), and steel fibers [1]. The materials used in the production of this material typically result in a high-cost product, however, by using local materials, the cost can be reduced significantly.

Over the last several years, a research project in collaboration with the New Mexico Department of Transportation (NMDOT) has investigated the feasibility of incorporating UHPC into bridge design in the State. Through the findings of a comprehensive literature and historical application review, trial designs, and cost analyses on typical prestressed concrete bridges using UHPC, it was determined that UHPC could potentially benefit design, construction, maintenance, and the lifespan of structures [2]. Optimized mixture proportions and a curing regimen for an UHPC that uses materials local to New Mexico were developed that meet the strength and durability design requirements of the NMDOT [3]. The creep and shrinkage behavior of the local UHPC has been investigated and Special Provisions have been developed for the implementation of UHPC in bridge design. To verify the design and behavior of UHPC girders, large scale flexural tests were conducted. Using the results of the research Bridge 9706, a two simple-span structure, was designed and constructed as the first UHPC bridge in New Mexico. One span of the bridge used UHPC and the other used high performance concrete (HPC). Bridge 9706 is currently being tested and monitored to investigate the performance of UHPC compared to HPC. Using the data gathered from the bridge, analytical models are being developed to further investigate the behavior Bridge 9706 and improve design methods for UHPC.

UHPC offers many advantages as its improved material and durability properties allow for smaller structural members to be used in designs, longer spans on bridges, and greatly increase the lifespans of structures [4]. It also reduces maintenance activities, maintenance costs, and life-cycle costs of concrete structures. With UHPC being a relatively new material, there are not many working professionals with experience or significant knowledge of the mixing, casting, curing, and testing of UHPC. Furthermore, many of the projects incorporating UHPC in their design have been in collaboration with researchers. Recently, testing standards became available for UHPC, however, there are currently no design codes that incorporate the material properties of UHPC. These issues have created a lack-of-knowledge in regards to the incorporation of UHPC with other materials in projects. As has been noted, emerging technologies are often difficult to get adopted due to a lack of dissemination, even when the technology offers many advantages [5]. Therefore, to disseminate the results of almost a decade of research and encourage the use of UHPC, workforce development and training seminars are necessary. Working with the NMDOT, the first of two workforce development symposiums was planned and held in early 2018.

\footnotetext{
* Corresponding author: bweldon@,nmsu.edu
} 


\subsection{Workforce development symposium}

The UHPC Symposium was hosted by New Mexico State University (NMSU) on April 17 - 18, 2018 at the Las Cruces Convention Center in Las Cruces, New Mexico. The Symposium focused on defining UHPC, identifying benefits of UHPC, and sharing experiences with mixing, casting, and curing UHPC. Through the knowledge gained through the research program, presentations helped to demonstrate how UHPC can be incorporated into precast facilities with little to no changes made to the facility or typical casting procedures. Furthermore, through the design of test specimens and the girders for Bridge 9706, engineers were exposed to how the improved properties of UHPC were incorporated into structural designs. Results of small- and large-scale testing were also presented to demonstrate the difference in behavior of typical high performance concrete and UHPC. Finally, hands-on demonstrations were provided to allow individuals to see the difference in the material from traditional concretes.

Working closely with the NMDOT, topics, speakers, advertising, and the venue were determined to ensure the needs of the State were met. While the Symposium was tailored for the needs of the NMDOT, contractors, designers, and suppliers were also encouraged to attend. Attendance to the UHPC Symposium was requested through the use of personal invitations, as well as, a save-the-date announcement. These invitations and announcements were sent to contractors, designers, suppliers, ready-mix companies, and researchers throughout the state of New Mexico and the surrounding regions. Professors and graduate students at New Mexico State University were also invited and encouraged to attend the Symposium. The save-the-date announcement that was sent out can be seen in Figure 1. To encourage a more diverse audience, the Symposium was offered free of charge and held the day prior to NM TransCon, New Mexico's Premier Transportation and Construction Conference. This conference is sponsored by NMSU College of Engineering, the NMSU Department of Civil Engineering, NMDOT, Associated Contractors of New Mexico, the American Planning Association - New Mexico Chapter, and the American Council of Engineering Companies - New Mexico.

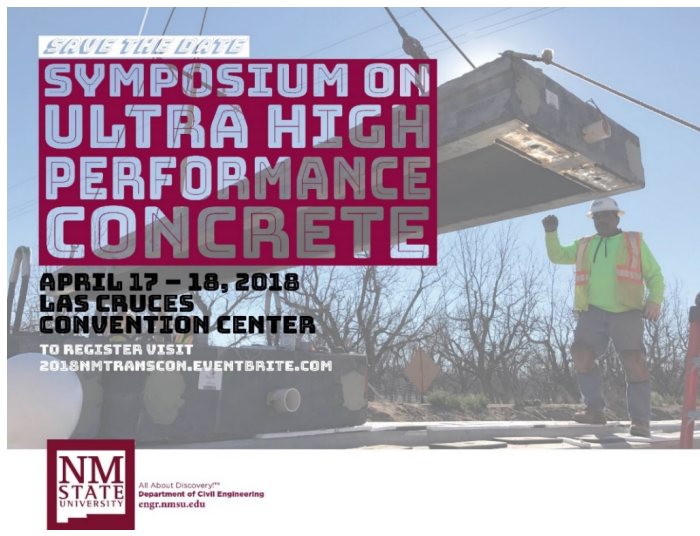

Fig. 1. UHPC symposium save-the-date announcement.

\subsection{UHPC symposium}

At the UHPC Symposium there were 11 presentations given over two days by professors, graduate students, and working professionals that discussed what UHPC is, its benefits, research that is currently in progress, how to test the material, and how engineers can use UHPC in their future designs. The list of presentations and speakers is provided below. Pictures from the presentations given at the Symposium can be found in Figure 2.

\subsubsection{April 17, 2018}

- Introduction - Lakshmi Reddi, Dean of the College of Engineering, NMSU; David Jauregui, Department Head, Civil Engineering, NMSU

- What is UHPC? - Brad Weldon, NMSU

- Upcoming NMDOT UHPC Projects - Kathy Crowell, NMDOT

- Research at NMSU - Feasibility to Implementation - Craig Newtson, NMSU

- Large Scale Testing - Michael J. McGinnis, University of Texas at Tyler

- Designing with UHPC - Danton Bean, HDR; Ben Najera, NMDOT

- Implementation in a Precast Plant - Mark Manning, NMSU

- Construction and Load Testing of Bridge 9706 Mark Manning, NMSU; Chris Kennedy, NMSU; and Alain Cuaron, NMSU

- UHPC Projects in New Mexico - Kathy Crowell, NMDOT

\subsubsection{April 18, 2018}

- Introduction - Brad Weldon, NMSU

- Overlay Project - Tran-SET, Craig Newtson, NMSU; Kathy Crowell, NMDOT

- Hands-on Demonstrations at the Structural Systems and Material Testing Laboratory at NMSU - Led by NMSU Graduate Students

On the second day of the UHPC Symposium, graduate students at NMSU assisted in demonstrating the mixing, casting, and testing procedures of UHPC to the attendees at the Structural Systems and Material Testing Laboratory (SSMTL) at the conclusion of the presentations. Pictures of these demonstrations can be found in Figure 3 and Figure 4.

At the UHPC Symposium, there were over 30 attendees including representatives of the NMDOT Materials Sections, NMDOT Bridge Bureau, NMDOT District Offices, regional contractors, design engineers, researchers (including international colleagues from Mexico), suppliers, and ready-mix concrete producers. 
(a)

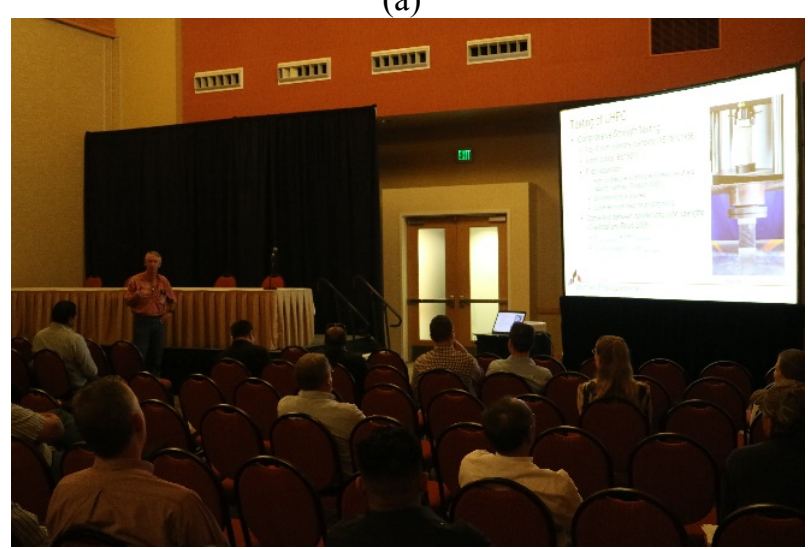

(b)

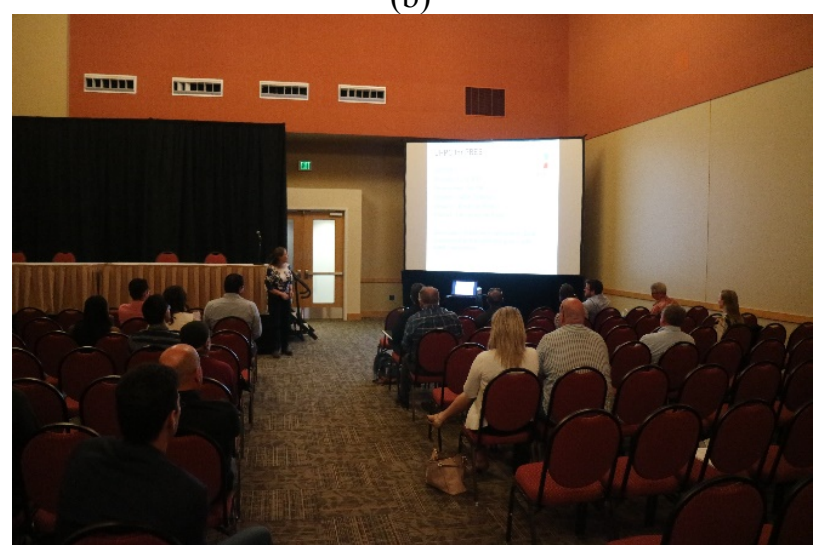

Fig. 2. UHPC symposium presentations.

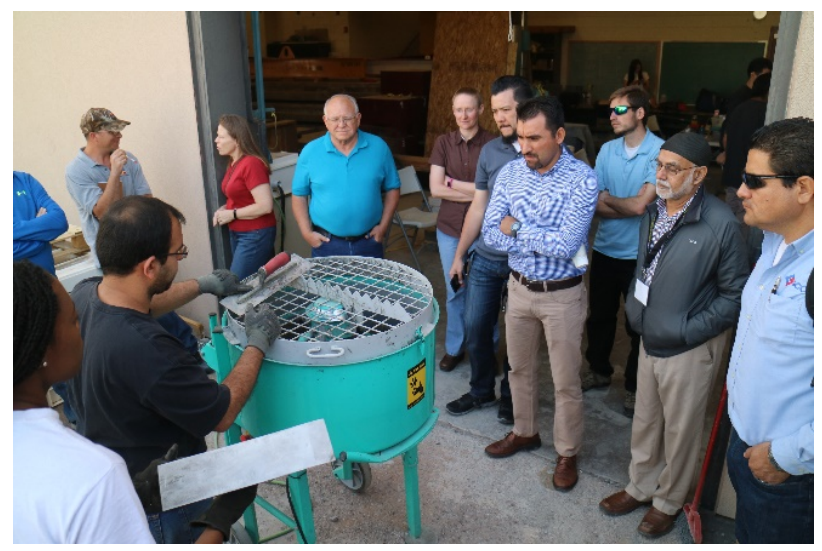

Fig. 3. Demonstration of proper UHPC mixing techniques.

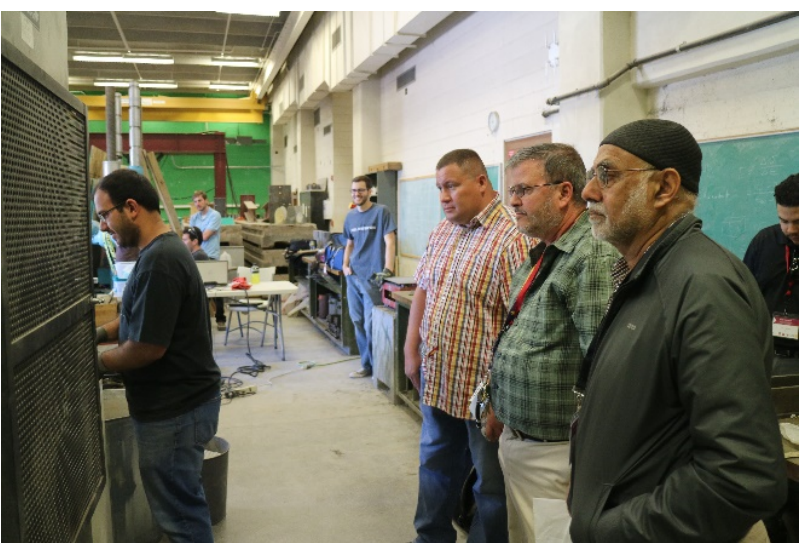

\section{Future needs}

As UHPC continues to emerge and new applications are introduced, there will continue to be a need for a platform to disseminate the knowledge and provide training. Following the successful completion of the first UHPC Symposium held in New Mexico, meetings were held with NMDOT and regional contractors to identify additional needs. Further training has been requested by the NMDOT and contractors to provide guidance on how to mix, place, and cure UHPC for different applications, many focused on upcoming NMDOT projects. Therefore, a second UHPC Symposium is currently being planned for early January 2019 and will be hosted by the NMSU Department of Civil Engineering in Albuquerque, New Mexico. This symposium will feature topics geared toward educating professionals on how to incorporate UHPC into designs and how to work with the material to ensure success. It will also satisfy the need of training for professionals to understand proper mixing, placement, curing, and testing techniques for this material. The target audience of the second symposium that will be invited to attend are the NMDOT, contractors, other regional DOT's, contractors, suppliers, researchers, and students.

\section{Conclusions}

The first of two workforce development symposiums on UHPC was successfully held in April 2018. Over a twoday period, information on UHPC was provided through presentations, hands-on demonstrations, and question and answer periods. With the NMDOT incorporating UHPC into more upcoming project, additional training needs are required, and therefore, a second symposium is curing in the planning stages for early 2019. The second symposium will focus on the training needs for contractors for an upcoming bridge rehabilitation that will implement the findings from the recent Tran-SET project on UHPC developed with local materials for overlays. As NMDOT projects continue to evolve, future research will be developed to address the needs of the State and provide the guidance and information necessary to ensure the success of the projects.

The authors would like to thank Tran-SET for funding this project, the New Mexico Department of Transportation for their continued support of UHPC research and collaboration, and BASF Chemical Company for their donations of silica fume and admixtures. Additionally, the authors would like to thank the following organizations and individuals for their contributions to the UHPC Symposium: NMSU College of Engineering; NMSU Department of Civil Engineering; Patricia Sullivan and Tracey O'Neil, Engineering New Mexico Resource Center; Virgil Valdez, NMDOT Research Bureau; Ray Trujillo, Ben Najera, and Kathy Crowell, NMDOT Bridge Bureau; Danton Bean, HDR; Michael McGinnis, University of Texas at Tyler; and NMSU Graduate Students: Ahmed AlBasha, Alain Cuaron, Chris Kennedy, Elsy Flores, Mark Manning, William Toledo; Turki Alahmari; Lulwa Al-Yahya; and Leticia Davila.

Fig. 4. Demonstration of testing for UHPC. 


\section{References}

1 Graybeal, B. (2006). Material Property Characterization of Ultra High Performance

Concrete. FHWA-HRT-06-103, Turner-Fairbank Highway Research Center, McLean, VA, 2006.

2 Weldon, B.D., Jauregui, D.V., Newtson, C.M., Taylor, C.W., Montoya, K.F., Allena, S., Muro, J., Tahat, M., Lyell, E., and Visage, E.T. (2009). Feasibility Analysis of Ultra High Performance Concrete for Prestressed Concrete Bridge Applications. Contract Number NM09MSC-01, New Mexico State University, Las Cruces, NM.

3 Weldon, B., Jauregui, D., Newtson, C., Montoya, K., Allena, C., Taylor, S., Muro, J., Tahat, M., Lyell, E., and Visage, E.T. (2012). Feasibility Analysis of Ultra High Performance Concrete for Prestressed Concrete Bridge Applications - Phase II. NMDOT Report No. NM09MSC-01, New Mexico State University, Las Cruces, NM.

4 Ahlborn, T.M., Li Misson, D., Peuse, E.J., Gilbertson, C.G. (2008). Durability and Strength

Characterization of Ultra-High Performance Concrete under Variable Curing Regimes. The Second International Symposium on Ultra High Performance Concrete, Kassel, Germany, 197-204.

5 Everett M. Rogers, Diffusion of Innovations, Fifth Edition 2003, Free Press, New York, 221 